\title{
Erratum: High-temperature behavior of supported graphene: Electron-phonon coupling and substrate-induced doping [Phys. Rev. B 86, 161402(R) (2012)]
}

\author{
Søren Ulstrup, Marco Bianchi, Richard Hatch, Dandan Guan, Alessandro Baraldi, Dario Alfè, \\ Liv Hornekær, and Philip Hofmann \\ (Received 9 May 2016; published 6 June 2016)
}

DOI: 10.1103/PhysRevB.93.239901

It has recently come to our attention that the code containing the fitting function shown in Eqs. (1) and (2) of the original paper contained several errors that significantly affect the order of magnitude of the extracted electron-phonon coupling strength $(\lambda)$ for graphene and the correlation of $\lambda$ with the effective Debye temperature $\left(\Theta_{D}\right)$. In Figs. E1(a) and E1(b) we present revised versions of Figs. 1(d) and 1(e) of our original paper with fits obtained using a corrected code.

For the fitted function in Fig. E1(a) we have used a fixed binding energy of $480 \mathrm{meV}$. This value is an average based on the binding energy range where we have extracted $\Sigma^{\prime \prime}(250-550 \mathrm{meV}$ below the Dirac point) and taking into account the shift of the Dirac point shown in Fig. 1(f) of our original paper. In our fit in Fig. E1(a) we have used an estimate of $50 \mathrm{meV}$ for the offset $\Sigma_{0}^{\prime \prime}$ to $\Sigma^{\prime \prime}$ due to electron-electron and electron-defect scattering, based on typical values of the slope and linewidth at the Fermi level of graphene, where the electron-phonon contribution is small. If $\Sigma_{0}^{\prime \prime}$ is taken as a free parameter, we obtain the correlation between $\lambda$ and $\Theta_{D}$ shown in Fig. E1(b). A good fit can only be obtained for a physically reasonable Debye temperature $\left(\Theta_{D} \geqslant 1268 \mathrm{~K}\right)$, consistent with our conclusion in the original paper. However, the extracted value of $\lambda$ is in the range between 0.5 and 1 , which is a typical value expected for metals and superconductors [1], rather than virtually zero as we originally concluded. If we instead fix $\Sigma_{0}^{\prime \prime}$ to our estimate of $50 \mathrm{meV}$, we obtain a single optimum for the fit as shown in Fig. E1(c). In this case, we obtain $\lambda=0.562$, which is much higher than we originally anticipated and also an order of magnitude larger than the value obtained from a linewidth analysis close to the Fermi energy of only lightly doped graphene [2].

Note that the error in the analysis does not imply a similarly large $\lambda$ near the Fermi level for this system. We rather attribute the high $\lambda$ to the average binding energy of $480 \mathrm{meV}$ where our analysis is carried out. The phase space for the electron-phonon coupling is not restricted in this case, unlike for the situation close to the Fermi energy. Our analysis here cannot be carried out close to the Fermi energy due to the strong spectral changes in this region and due to the difficulty of fitting the linewidth when the two linear bands are close. Finally, we emphasize that this error does not affect the second part of the original paper which deals with the substrate-induced doping.

We thank Nicki Hinsche for bringing the error in the fit to our attention.

(a)

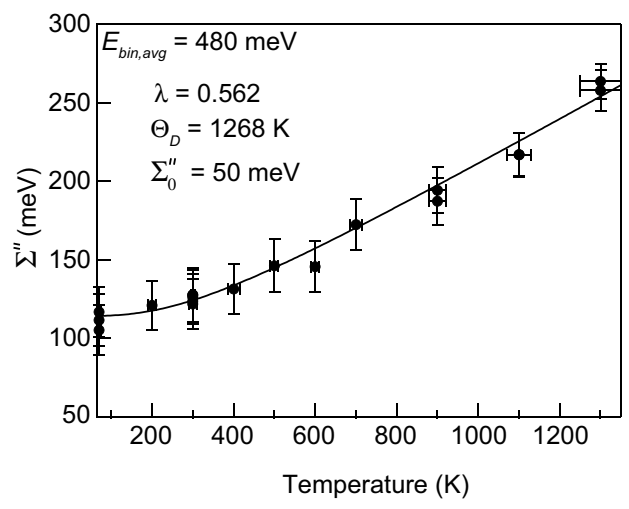

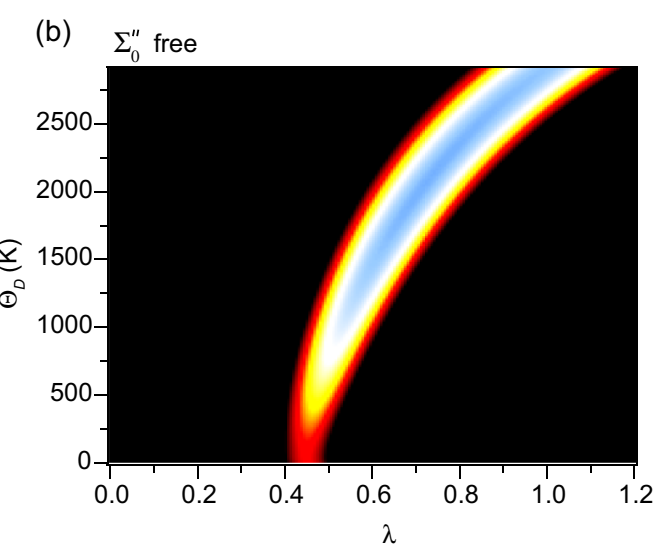

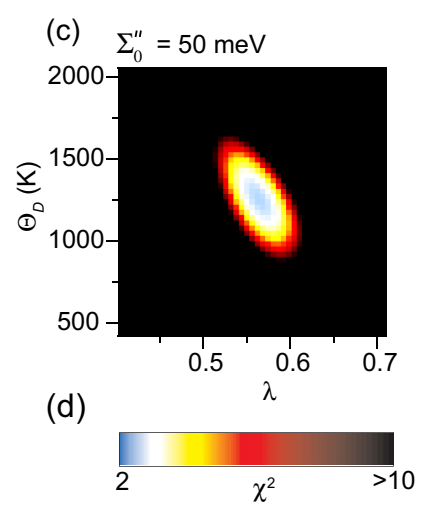

FIG. E1. (a) Imaginary part of the self-energy reproduced from Fig. 1(d). The solid line is the new fit result with the given parameters. (b) Revised map of $\chi^{2}$ for the fit in (a) as a function of $\Theta_{D}$ and $\lambda$. The value of $\Sigma_{0}^{\prime \prime}$ is here taken as a free fit parameter as in Fig. 1(e) of the original paper. (c) Map of $\chi^{2}$ as in (b) but with a fixed $\Sigma_{0}^{\prime \prime}$ value of $50 \mathrm{meV}$. (d) Scale bar for $\chi^{2}$ corresponding to (b) and (c).

[1] P. Hofmann, I. Y. Sklyadneva, E. D. L. Rienks, and E. V. Chulkov, Electron-phonon coupling at surfaces and interfaces, New J. Phys. 11, 125005 (2009).

[2] J. C. Johannsen, S. Ulstrup, M. Bianchi, R. Hatch, D. Guan, F. Mazzola, L. Hornekær, F. Fromm, C. Raidel, T. Seyller, and P. Hofmann, Electron-phonon coupling in quasi-free-standing graphene, J. Phys.: Condens. Matter 25, 094001 (2013). 